\title{
Angewandte Geostatistik in der landwirtschaftlichen Versuchsplanung
}

\author{
Herbert Schwarz \\ Institut für Mathematik und Angewandte Statistik \\ Universität für Bodenkultur
}

\begin{abstract}
Zusammenfassung: In der landwirtschaftlichen Versuchsplanung tritt oft auch eine räumliche und/oder eine zeitliche Komponente auf. Bei Feldversuchen mit raumbezogenen Daten kann die Variation zwischen Messungen bei einer bestimmten Distanz mit Hilfe des Semivariogramms modelliert werden. Anhand eines praktischen Beispiels wird bei einem 50 mal $100 \mathrm{~m}$ großen Versuchsfeld in einem 7 mal 7 m Raster der Aufbau des Bodens, der sich bis zu einer Tiefe von $120 \mathrm{~cm}$ in drei unterschiedliche Horizonte gliedert, untersucht. Für die Strukturanalyse im obersten Bodenhorizont wird ein geschachteltes Variogrammodell mit Nuggeteffekt, einer sphärischen und zwei gauß'schen Komponenten, bei zonaler und geometrischen Anisotropie angepaßt.
\end{abstract}

\begin{abstract}
In agricultural experimental design a spatial or temporal component can often be observed. In the spatial analysis of field trials the variation between measurements at a certain distance can be modelled using the semivariogram. In a practical experiment in a 50 by $100 \mathrm{~m}$ field the structure of the soil is investigated in a 7 times $7 \mathrm{~m}$ grid to a depth of $120 \mathrm{~cm}$. Within that depth the soil is subdivided into three levels. For the structure-analysis of the topmost level, a combined variogram model, including a nugget variance, a spherical and two gaussian components, is used, modelling a mixture of geometric and zonal anisotropies.
\end{abstract}

Schlüsselwörter: Geostatistik, Strukturanalyse, Variogramm.

\section{Einleitung}

In der landwirtschaftlichen Versuchsplanung treten oft auch räumliche und/oder zeitliche Beziehungen auf, die bei einer richtigen statistischen Versuchsauswertung auch berücksichtigt werden. Bei Feldversuchen mit raumbezogenen Daten kann die Variation zwischen Messungen bei einer bestimmten Distanz mit Hilfe des Semivariogramms modelliert werden. Die Kenntnis der räumlichen Struktur einer oder mehrerer Variable kann als wesentliche Information für die statistische Datenanalyse der Feldversuche, für die Anlage neuer Versuche, für die Meßnetzplanung und Meßnetzoptimierung und für die räumliche Interpolation mit Hilfe des Krigen eingesetzt werden. 
Im Rahmen dieser Arbeit werden raumbezogene Daten, die untersuchte Variable ist die Bodentiefe des obersten Bodenhorizontes, analysiert. Der untersuchte Boden ist ein tiefgründiger, fruchtbarer Schwarzerdeboden, der sich bis zu einer Tiefe von $120 \mathrm{~cm}$ in drei unterschiedliche Horizonte gliedert. Der oberste Horizont ist der sogenannte AHorizont, dabei handelt es sich um den fruchtbaren, humushältigen Oberboden, der ungefähr bis $\mathrm{zu}$ einer Tiefe von $40 \mathrm{~cm}$ vorkommt. Die Tiefe des obersten Bodenhorizontes wurde in einem 50 mal 100 m großen Versuchsfeld in einem 7 mal 7 m Raster untersucht. Die Erhebungen wurden im Frühjahr 1995 im Raum Großenzersdorf durchgeführt.

\section{Strukturanalyse}

Das Semivariogramm dient als eines der wichtigsten Werkzeuge in der Strukturanalyse der räumlichen Verteilung regionalisierter Variable. Dazu stellt das empirische Variogramm ein wesentliches Werkzeug für eventuelle weitere Studien dar. Nach WEBSTER und OLIVER (1990) sind Semivariogramme normalerweise charakterisiert durch den Klumpeneffekt $C_{0}$, die Reichweite oder den Einflußbereich $a$ und den Schwellenwert $C$. Für die Berechnung und graphische Darstellung der Semivariogramme findet das Programm GEOSAN von DUTTER (1995b) Verwendung.

Nach JOURNEL und HuiJBREGTS (1978) ist das experimentelle Variogramm nur bis zu einer Distanz kleiner gleich der halben Größe der Untersuchungsfläche interpretierbar und zur Berechnung eines Variogrammwertes sollen mindestens 30 bis 50 Paare Verwendung finden. Werden Stichproben auf einem regelmäßigen oder pseudoregelmäßigen Gitter genommen, kann auch weniger als die Hälfte des untersuchten Bereiches als Einflußbereich genommen werden. Das experimentelle Variogramm wird für einen Bereich von $50 \mathrm{~m}$ und $30 \mathrm{~m}$ in den beiden Hauptrichtungen dargestellt, wobei ein Variogrammwert von mindestens 40 Paaren berechnet wird (Tabelle 1 und Tabelle 2).

Für die Berechnung richtungsabhängiger Semivariogramme ist der Toleranzwinkel so klein als möglich zu nehmen, um eine verschwommene Anisotropie zu vermeiden, die aus der Kombination von Distanzen verschiedener Richtungen entstehen. Allerdings kann ein zu schmaler Toleranzwinkel eine falsche Variogrammschätzung bewirken, vor allem dann, wenn zu wenige Distanzpaare in einer Richtung vorliegen, um eine geeignete Beschreibung der Struktur zu liefern. Nach ISAAKS und SRIVASTAVA (1989) besteht die beste Vorgangsweise darin, mehrere Toleranzwinkel für die Variogrammberechnung zu probieren, um dann den kleinsten, der noch gute Resultate liefert, auszuwählen. Für die Berechnung richtungsabhängiger Variogramme erscheint im hier vorliegenden Beispiel ein Toleranzwinkel von $45^{\circ}$ am geeignetsten.

Wenn die Anisotropieachsen nicht mit den Hauptrichtungen der Daten zusammenfallen, muß eine Koordinatentransformation durchgeführt werden. Die Anisotropieachsen fallen mit den Hauptrichtungen der Daten in der Regel nicht zusammen, die Orien- 
tierung der Datenachsen ist zufällig und die Orientierung der Anisotropieachsen hängt mit dateneigenen Phänomenen zusammen.

Tabelle 1: Empirisches Variogramm in der Hauptrichtung $15^{\circ}$

VARIABLE: Bodentiefe von Horizont A

$\begin{array}{lcc}\text { NUMBER OF CONES: } 1 & \\ \text { CONE } & \text { ANGLE } & \text { TOLERANCE } \\ (1) & 15 & 45\end{array}$

NUMBER OF LAGS: $\quad 19$

TOTAL NUMBER OF PAIRS: 2712

MIN. NUMBER OF PAIRS PER LAG: $\quad 40$

$\begin{array}{cccccc}\text { LAG } & \text { MIN.DIS. } & \text { MAX.DIS. } & \text { AVG.DIS. } & \text { PAIRS } & \text { VALUE } \\ 1 & 7.0000 & 7.0000 & 7.0000 & 102 & 38.525 \\ 2 & 9.8995 & 9.8995 & 9.8995 & 91 & 64.082 \\ 3 & 14.000 & 14.000 & 14.000 & 95 & 40.737 \\ 4 & 15.652 & 15.652 & 15.652 & 167 & 64.072 \\ 5 & 19.799 & 21.000 & 20.464 & 159 & 66.503 \\ 6 & 22.136 & 22.136 & 22.136 & 157 & 63.350 \\ 7 & 25.239 & 25.239 & 25.239 & 127 & 75.303 \\ 8 & 28.000 & 28.000 & 28.000 & 81 & 67.605 \\ 9 & 28.862 & 29.698 & 29.099 & 201 & 69.075 \\ 10 & 31.305 & 31.305 & 31.305 & 122 & 83.541 \\ 11 & 35.000 & 35.000 & 35.000 & 170 & 61.412 \\ 12 & 35.693 & 37.696 & 36.611 & 240 & 87.835 \\ 13 & 39.598 & 39.598 & 39.598 & 41 & 37.012 \\ 14 & 40.817 & 42.579 & 41.894 & 264 & 71.513 \\ 15 & 44.272 & 44.822 & 44.496 & 167 & 58.231 \\ 16 & 46.957 & 46.957 & 46.957 & 83 & 76.367 \\ 17 & 49.000 & 49.497 & 49.345 & 189 & 78.937 \\ 18 & 50.478 & 50.961 & 50.778 & 140 & 62.482 \\ 19 & 53.310 & 54.672 & 53.815 & 116 & 66.871\end{array}$

Nach ISAAKS und SRIVASTAVA (1989) kann die Bestimmung der Anisotropieachsen auch mit Hilfe des Sterndiagramms erfolgen. Dabei müssen die Richtungen mit maximaler und minimaler Reichweite graphisch gefunden werden. Diese Richtungen entsprechen den Anisotropieachsen. Dabei werden die jeweiligen Werte ermittelt und in ein Sterndiagramm eingetragen. Diesem Sterndiagramm wird eine Ellipse angepaßt, deren Hauptachsen den Anisotropieachsen entsprechen. 
Tabelle 2: Empirisches Variogramm in der Hauptrichtung $105^{\circ}$

VARIABLE: Bodentiefe von Horizont A

NUMBER OF CONES: 1

CONE ANGLE

(1)

105

TOLERANCE

45

NUMBER OF LAGS:

11

TOTAL NUMBER OF PAIRS:

1250

MIN. NUMBER OF PAIRS PER LAG:

40

$\begin{array}{cccccc}\text { LAG } & \text { MIN.DIS. } & \text { MAX.DIS. } & \text { AVG.DIS. } & \text { PAIRS } & \text { VALUE } \\ 1 & 7.0000 & 7.0000 & 7.0000 & 96 & 44.057 \\ 2 & 9.8995 & 9.8995 & 9.8995 & 90 & 58.267 \\ 3 & 14.000 & 14.000 & 14.000 & 82 & 98.689 \\ 4 & 15.652 & 15.652 & 15.652 & 153 & 90.121 \\ 5 & 19.799 & 21.000 & 20.387 & 139 & 106.37 \\ 6 & 22.136 & 22.136 & 22.136 & 128 & 105.81 \\ 7 & 25.239 & 25.239 & 25.239 & 124 & 92.298 \\ 8 & 28.000 & 28.000 & 28.000 & 54 & 90.556 \\ 9 & 28.862 & 29.698 & 29.151 & 156 & 92.244 \\ 10 & 31.305 & 31.305 & 31.305 & 95 & 73.432 \\ 11 & 35.000 & 35.000 & 35.000 & 133 & 108.56\end{array}$

Eine andere Möglichkeit zur Bestimmung der Anisotropieachsen ergibt sich aus der rechnerischen Ermittlung, abgewandelt nach DUTTER (1995a). Dabei wird von zwei Hauptrichtungen, $0^{\circ}$ und $90^{\circ}$, und einer dazwischenliegenden Richtung, $45^{\circ}$, ausgegangen. Die dazugehörenden Einflußbereiche seien $r_{1}, r_{2}, r_{3}$. Die drei Punkte $\left(x_{1}, 0\right), \quad\left(0, y_{2}\right)$ und $\left(x_{3}, y_{3}\right)$ mit $x_{1}=r_{1}, y_{2}=r_{2}$ und $x_{3}=r_{3} \cos \alpha, \quad y_{3}=r_{3} \sin \alpha$ definieren folgende Ellipse mit dem Zentrum $\left(\begin{array}{l}0 \\ 0\end{array}\right): \frac{x^{2}}{a^{2}}+2 \frac{x y}{b}+\frac{y^{2}}{c^{2}}=1$

Die Parameter a, b und c werden durch Einsetzen der drei Punkte in die Ellipsengleichung berechnet:

$$
\begin{aligned}
\frac{\mathrm{x}_{1}^{2}}{\mathrm{a}^{2}} & =1 \\
\frac{\mathrm{y}_{2}^{2}}{\mathrm{c}^{2}} & =1 \\
\frac{\mathrm{x}_{3}^{2}}{\mathrm{a}^{2}}+2 \frac{\mathrm{x}_{3} \mathrm{y}_{3}}{\mathrm{~b}}+\frac{\mathrm{y}_{3}^{2}}{\mathrm{c}^{2}} & =1
\end{aligned}
$$




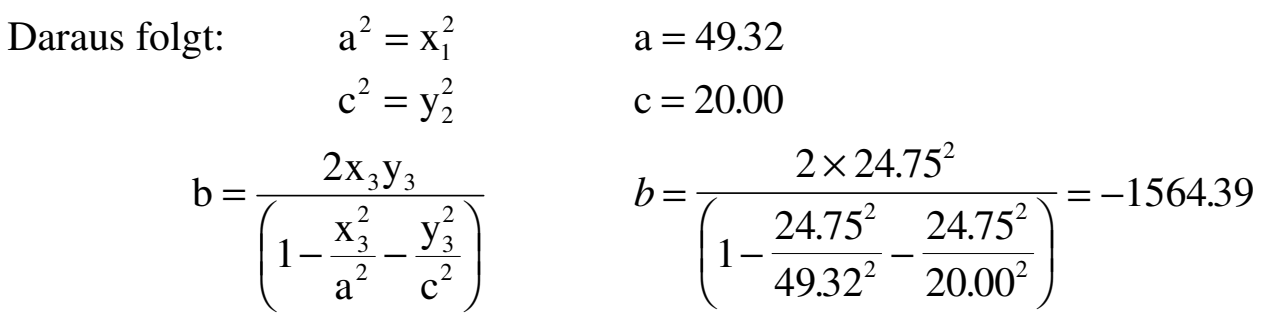

Die Berechnung des Drehwinkels zwischen den Hauptachsen der Ellipse, die den Anisotropieachsen entsprechen, und den Hauptachsen der Daten erfolgt durch:

$$
\delta=\frac{1}{2} \arctan \frac{\frac{2}{b}}{\frac{1}{a^{2}}-\frac{1}{c^{2}}}=15.73
$$

Daraus werden die Anisotropieachsen mit den Richtungen $15^{\circ}$ und $105^{\circ}$ abgeleitet. Die Länge der Hauptachsen der Ellipse sind $a_{1}=65.74$ und $b_{1}=19.32$. Sie werden aus dem Schnittpunkt der Geraden $\left(\begin{array}{l}x \\ y\end{array}\right)=\lambda\left(\begin{array}{l}\cos \delta \\ \sin \delta\end{array}\right)$ und ihrer Normalen mit der Ellipse berechnet.

Die Drehmatrix für die Rotation der Datenhauptachsen in die anisotrope Hauptachsenposition lautet:

$$
R=\left(\begin{array}{rr}
\cos \delta & \sin \delta \\
-\sin \delta & \cos \delta
\end{array}\right)
$$

Nach Bestimmung der Anisotropieachsen soll ein Modell gefunden werden, das die Veränderung sowohl in der Distanz als auch in der Richtung beschreibt. Eine Methode für die Kombination von Modellen verschiedener Richtungen in ein konsistentes Modell in allen Richtungen ist, eine Transformation $\mathrm{zu}$ finden, die alle gerichteten Variogramme zu einem Modell mit dem standardisierten Einflußbereich von 1 zusammenfaßt. Die Methode dabei ist, den Einflußbereich so zu transformieren, daß das standardisierte Modell einen Variogrammwert für eine bestimmte Distanz in einer bestimmten Richtung liefert, der ident ist mit dem Wert des urspünglichen gerichteten Variogramms.

Für die Strukturanalyse im obersten Horizont wird ein geschachteltes Variogramm mit Nuggeteffekt bei zonaler und geometrischer Anisotropie modelliert, da Varianz und der Anstieg der Variogramme richtungsabhängig sind. Der Ausdruck $\vec{h}$ des Variogramms ist vektorartig, besteht aus dem absoluten Betrag $|h|$ und der Richtung $\alpha$. Das Modell lautet:

$$
\gamma(\vec{h})=C_{0}+\gamma_{1}\left(\vec{h}_{1}\right)+\gamma_{2}\left(\vec{h}_{2}\right)+\gamma_{3}\left(\vec{h}_{3}\right)
$$

Die Nuggetvarianz $C_{0}=20$. 
Die zweite Komponente des Modells ist ein isometrisches Sphärisches Modell nach Matheron mit einem Einflußbereich $\mathrm{a}_{1}=10$ und einem Schwellenwert $C_{1}=30$.

$$
\begin{array}{ll}
\gamma_{1}\left(\vec{h}_{1}\right)=C_{1}\left[\frac{3}{2} h_{1}-\frac{1}{2} h_{1}^{3}\right] & \text { für } 0 \leq h_{1} \leq a_{1} \\
C_{1} & \text { für } h_{1} \geq a_{1} \\
\vec{h}_{1}=\left(\begin{array}{cc}
\frac{1}{a_{1}} & 0 \\
0 & \frac{1}{a_{1}}
\end{array}\right) \times\left(\begin{array}{l}
h_{x} \\
h_{y}
\end{array}\right) &
\end{array}
$$

Laut CRESSIE (1991) ist das Sphärische Modell gekennzeichnet durch ein lineares Verhalten im Ursprung. Die Tangente an das Variogramm, die durch den Ursprung geht, erreicht den Schwellenwert bei $2 / 3$ der Reichweite.

Als nächste Komponente wird ein Gauß’sches Modell mit Einflußbereich $a_{2}=19.32$ und einem Schwellenwert $C_{2}=50$ angepaßt:

$$
\begin{gathered}
\gamma_{2}\left(\overrightarrow{\mathrm{h}}_{2}\right)=\mathrm{C}_{2}\left[1-\mathrm{e}^{-\mathrm{h}_{2}^{2}}\right] \\
\vec{h}_{2}=\left(\begin{array}{ll}
0 & 1 \\
0 & 1
\end{array}\right) \times\left(\begin{array}{cc}
\frac{1}{a_{2}} & 0 \\
0 & \frac{1}{a_{2}}
\end{array}\right) \times\left(\begin{array}{rr}
\cos \delta & \sin \delta \\
-\sin \delta & \cos \delta
\end{array}\right) \times\left(\begin{array}{l}
h_{x} \\
h_{y}
\end{array}\right)=\left(\begin{array}{ll}
-0.0140 & 0.0498 \\
-0.0140 & 0.0498
\end{array}\right) \times\left(\begin{array}{l}
h_{x} \\
h_{y}
\end{array}\right)
\end{gathered}
$$

Mit dem Vektor $\overrightarrow{\mathrm{h}}_{2}$ wird der Vektor $\overrightarrow{\mathrm{h}}$ des ursprünglichen Koordinatensystems in das anisotrope Koordinatensystem transformiert, dessen Hauptrichtungen mit den Anisotropieachsen übereinstimmen.

Als letzte Komponente wird ebenfalls ein Gauß’sches Modell mit Einflußbereich $a_{3}=65.74$ und einem Schwellenwert $C_{3}=30$ angepaßt:

$$
\begin{gathered}
\gamma_{3}\left(\overrightarrow{\mathrm{h}}_{3}\right)=\mathrm{C}_{3}\left[1-\mathrm{e}^{-\mathrm{h}_{3}^{2}}\right] \\
\vec{h}_{3}=\left(\begin{array}{ll}
1 & 0 \\
1 & 0
\end{array}\right) \times\left(\begin{array}{cc}
\frac{1}{a_{3}} & 0 \\
0 & \frac{1}{a_{3}}
\end{array}\right) \times\left(\begin{array}{rr}
\cos \delta & \sin \delta \\
-\sin \delta & \cos \delta
\end{array}\right) \times\left(\begin{array}{l}
h_{x} \\
h_{y}
\end{array}\right)=\left(\begin{array}{ll}
0.0146 & 0.0041 \\
0.0146 & 0.0041
\end{array}\right) \times\left(\begin{array}{l}
h_{x} \\
h_{y}
\end{array}\right)
\end{gathered}
$$

Nach DutTER (1995a) ist das Gauß'sche Modell gekennzeichnet durch ein quadratisches Verhalten im Ursprung, das Modell hat im Ursprung die Steigung 0. Das Gauß'sche Modell erreicht den Schwellenwert nur asymptotisch, und zwar erreicht es $95 \%$ des Schwellenwertes beim $\sqrt{3}$-fachen Einflußbereich. 
Abbildung 1: Semivariogrammodell und empirisches Variogramm in Hauptrichtung $15^{\circ}$ Semi-variogram of $\mathrm{A}$

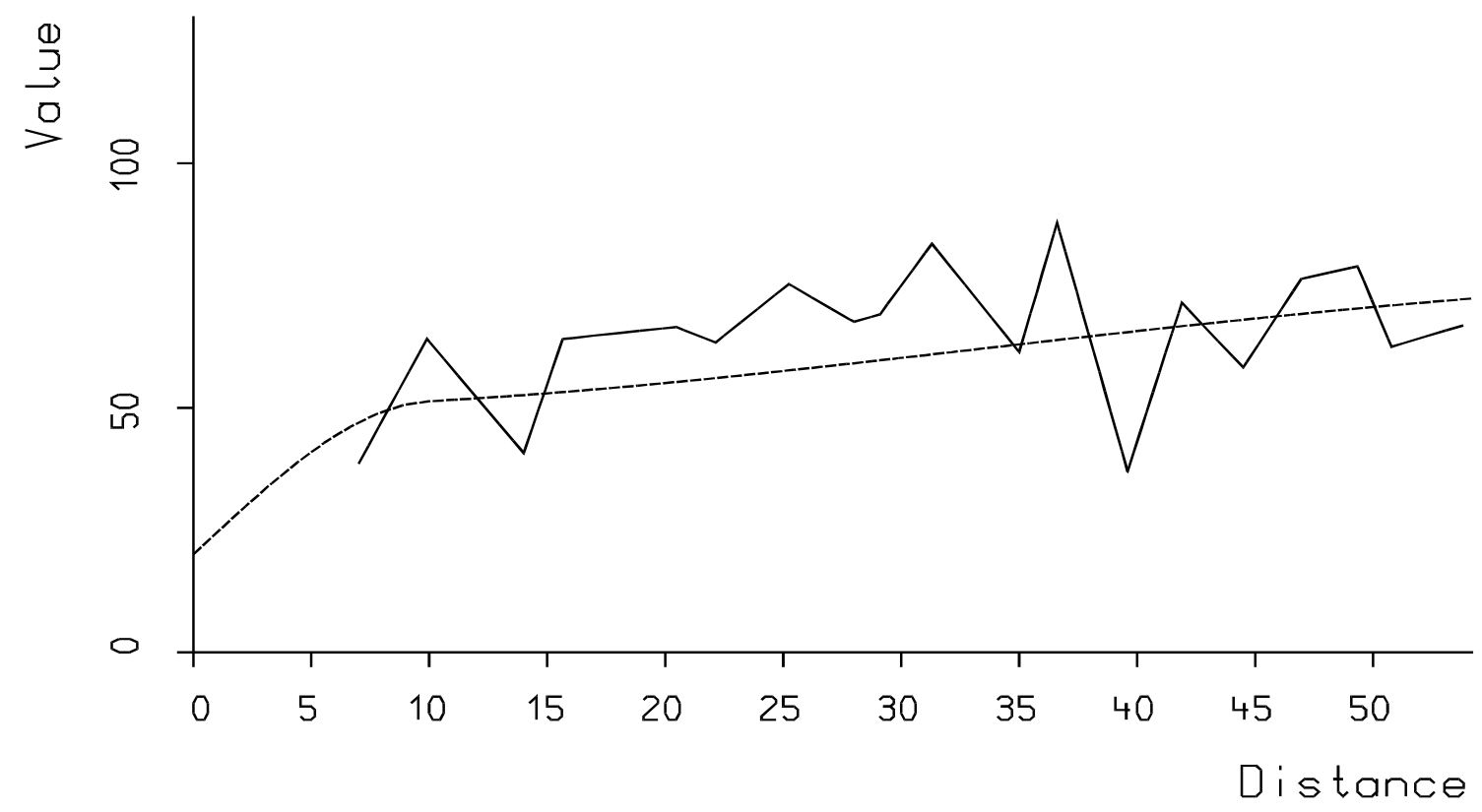
15 Model

Abbildung 2: Semivariogrammodell und empirisches Variogramm in Hauptrichtung $105^{\circ}$

Semi-variogram of $A$

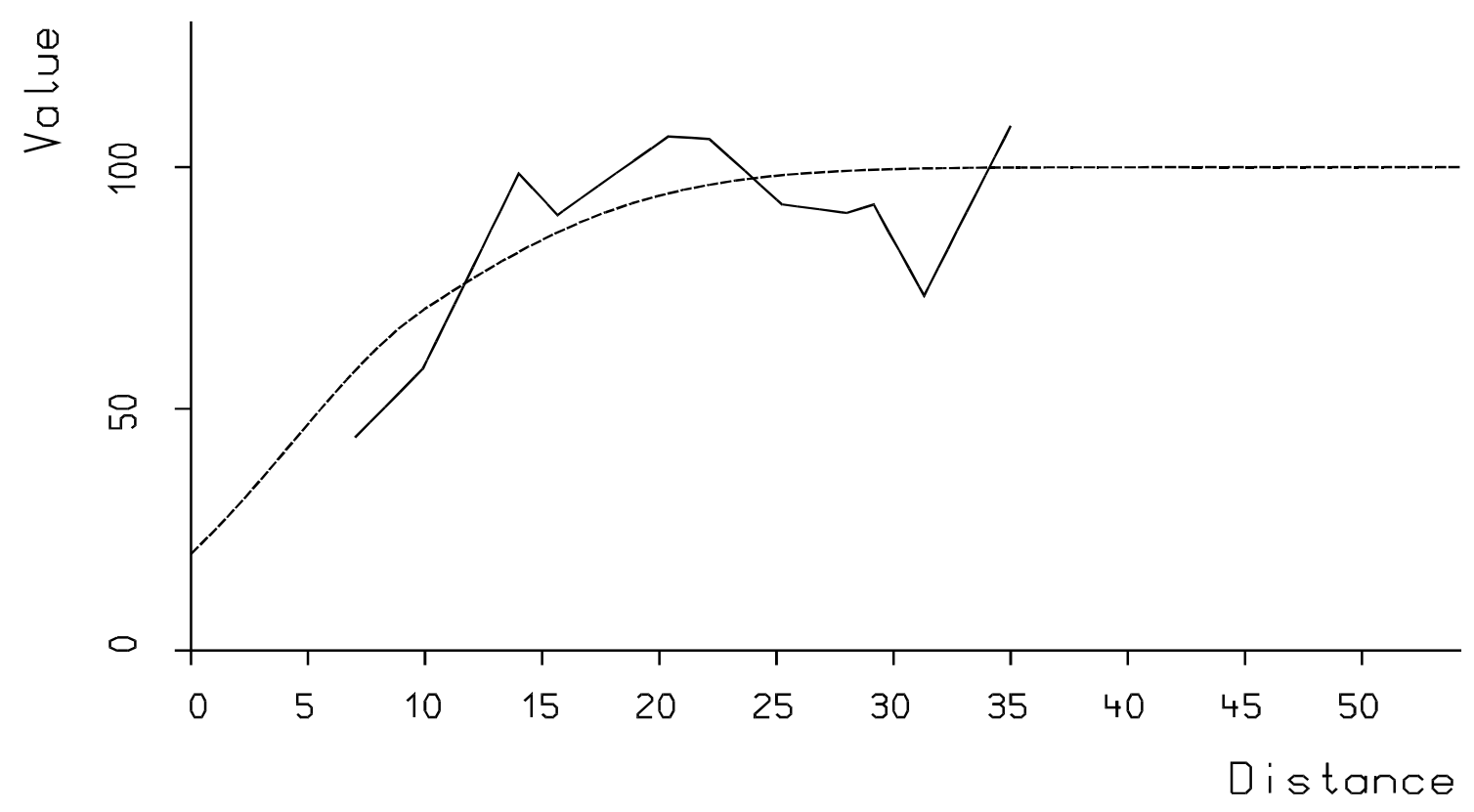


Das Modell wird einerseits für eine Serie von Distanzen entlang der Anisotropieachsen evaluiert (Abbildung 1 und Abbildung 2), um zu überprüfen, ob es die entsprechenden richtungsabhängigen Variogramme liefert. Darüberhinaus ist das Modell auch für mehrere willkürliche Richtungen zwischen den Anisotropieachsen berechnet und diese Werte mit dem Stichprobenvariogramm in der entsprechenden Richtung verglichen worden. Die erfolgreiche Evaluierung deutet auf die richtige Erkennung der Anisotropieachsen und auf die Verwendung des richtigen Modells hin.

Das gesamte Variogrammodell lautet:

$$
\text { für } \begin{aligned}
0<h \leq a_{1}: \gamma(\vec{h})=20 & +30 \times\left[\frac{3}{2}\left(\begin{array}{cc}
\frac{1}{10} & 0 \\
0 & \frac{1}{10}
\end{array}\right)\left(\begin{array}{c}
h_{x} \\
h_{y}
\end{array}\right)-\frac{1}{2}\left[\left(\begin{array}{cc}
\frac{1}{10} & 0 \\
0 & \frac{1}{10}
\end{array}\right)\left(\begin{array}{l}
h_{x} \\
h_{y}
\end{array}\right)\right]^{3}\right]+ \\
& +50 \times\left[1-e^{-\left[\left(\begin{array}{cc}
-0.0140 & 0.0498 \\
-0.0140 & 0.0498
\end{array}\right)\left(\begin{array}{l}
h_{x} \\
h_{y}
\end{array}\right)\right]^{2}}\right]+30 \times\left[1-e^{-\left[\left(\begin{array}{ll}
0.0146 & 0.0041 \\
0.0146 & 0.0041
\end{array}\right)\left(\begin{array}{l}
h_{x} \\
h_{y}
\end{array}\right)\right]^{2}}\right]
\end{aligned}
$$

für $h \geq a_{1}$ :

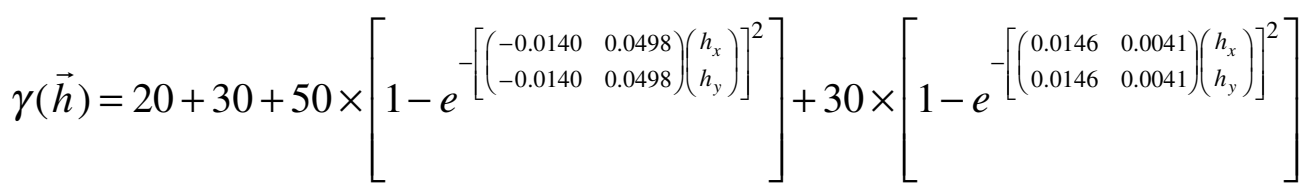

\section{Schlußfolgerungen}

Die räumliche Beziehung der Variable Mächtigkeit des obersten Bodenhorizontes wird durch ein geschachteltes Variogrammodell mit Nuggeteffekt, einer sphärischen und zwei gauß'schen Komponenten, bei zonaler und geometrischen Anisotropie beschrieben. Das anisotrope Verhalten der Variable Bodenmächtigkeit deutet darauf hin, daß sowohl die Varianz als auch die Covarianz richtungsabhängig sind.

Von der Art und dem Aufbau der Bodenhorizonte leiten sich die wesentlichen Eigenschaften des Bodens, wie zum Beispiel Wasserhaltefähigkeit, Bodenfruchtbarkeit und Nährstoffverfügbarkeit, ab. Die Kenntnis der räumlichen Struktur des Bodenaufbaus, in weiterer Folge auch die Kenntnis der bodenphysikalischen und bodenchemischen Kennwerten, kann als wesentliche Information für die statistische Datenanalyse der Feldversuche, für die Anlage neuer Versuche, für die Meßnetzplanung und Meßnetzoptimierung und für die räumliche Interpolation mit Hilfe des Krigen eingesetzt werden. Diese Themenbereiche sollen Gegenstand für zukünftige Arbeiten sein. 


\section{Literatur}

CRessie, N.A.C. (1991). Statistics for Spatial Data. New York: Wiley \& Sons.

DUTTER, R. (1995a). Geostatistik, Technische Universität Wien: Vorlesungsskriptum.

DUTTER, R. (1995b). GEOSAN - Program System for Geostatistical Analysis, Technische Universität Wien: Handbook.

ISAaKs, E.H., SRIVAStaVA, R.M. (1989). An Introduction to Applied Geostatistics. New York, Oxford: Oxford University Press.

Journel, A.G., HuiJBregts, C.J. (1978). Mining Geostatistics. New York: Academic Press.

Webster, R., Oliver, M.A. (1990). Statistical Methods in Soil and Land Resource Survey. New York, Oxford: Oxford University Press.

Adresse des Autors:

Univ.-Ass. DI. Dr. Herbert Schwarz

Institut für Mathematik und Angewandte Statistik

Universität für Bodenkultur

Gregor Mengelstr. 33, A-1180 Wien

E-Mail: schwarzh@mail.boku.ac.at

Tel. ++43-1-47654-5063

Fax. ++43-1-47654-5217 